\title{
Sprachliche und religiöse Probleme erschweren die Einstellung
}

\author{
Diabetespatienten aus einem anderen Kultur- bzw. Sprachraum brauchen besondere Auf- \\ merksamkeit. Besonderheiten der Lebensführung und der Ernährung müssen berücksichtigt \\ werden. Traditionen und Tabus können Auswirkungen auf die Stoffwechselkontrolle haben.
}

\begin{abstract}
Immer mehr Diabetiker beim Hausarzt haben einen Migrationshintergrund. Meist handelt es sich dabei heute noch um Typ-1-Diabetiker. Aber je länger Migranten in Deutschland leben, desto stärker passen sie sich an westliche Ernährungs- und Lebensbedingungen an mit der Folge, dass auch vermehrt ein Typ-2-Diabetes manifest wird, erklärte I.-C. Omumuh, Mainz.
\end{abstract}

\section{Verständnis ist gefragt}

Das Hauptproblem bei der Betreuung dieser Patienten sind ihre oft unzureichenden Sprachkenntnisse. Deshalb sind für die Beratung und Schulung fremdsprachliche Arbeitsmaterialien unverzichtbar. Sehr hilfreich sind Behandlungsbilderbücher mit gut erfassbaren Illustrationen. Soweit machbar, ist es sinnvoll, sprachkundige Angehörige einzubinden. Stehen solche nicht zur Verfügung, muss ein
Dolmetscher hinzugezogen werden. Wichtig ist auch, dass der Patient zu einer Diabetesberaterin ein Vertrauensverhältnis aufbauen kann. Vorteilhaft ist es, wenn eine der Beraterinnen selbst einen Migrationshintergrund hat. Grundsätzlich sollte man solchen Problempatienten mit Verständnis begegnen

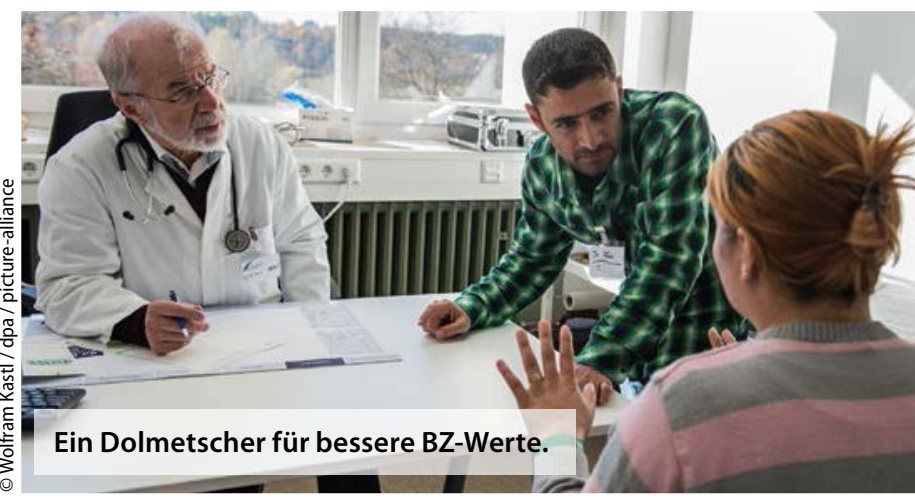
und sie nicht zu schnell als schulungsunfähig abqualifizieren.
Was tun im Ramadan? Besonders bei Frauen mit Schwangerschaftsdiabetes spielen auch religiöse Aspekte eine Rolle. Eine Insulintherapie wird oft nicht durchgeführt, weil fälschlicherweise angenommen wird, dass es sich dabei um Schweineinsulin handelt.

\section{Gemeinsame Sprache senkt den Blutzucker}

Eine Studie aus den USA bestätigt die Bedeutung einer funktionierenden Verständigung zwischen Arzt und Patient für eine gute Blutzuckerkontrolle. Lateinamerikanischen Diabetespatienten, die kaum Englisch verstanden, hatten ihren Blutzucker besser unter Kontrolle, wenn sie von einem ausschließlich Englisch zu einem auch Spanisch sprechenden Hausarzt wechselten.

Statt vorher $63 \%$ erreichten dann $74 \%$ von 418 Südamerikanern einen $\mathrm{HbA}_{1 \mathrm{c}}<8 \%$. Verglichen mit Patienten, die einen umgekehrten Arztwechsel absolviert hatten, erhöhte sich der Anteil derer mit guter Blutzuckereinstellung um $10 \%$, der Anteil mit schlechter Einstellung $\left(\mathrm{HbA}_{1 \mathrm{c}}>\right.$ 9\%) sank um 4\%. Der $\mathrm{HbA}_{1 c}$-Wert war im Mittel um 0,22 Prozentpunkte zurückgegangen.

Mit einem Dolmetscher-Service war es bereits in einer vorangegangenen Studie mit Latinos in den USA gelungen, den Anteil der Patienten mit schlechter Blutzuckereinstellung deutlich zu reduzieren.

- bs

- Parker MM et al. Association of Patient-Physician Language Concordance and Glycemic Control for Limited-English Proficiency Latinos With Type 2 Diabetes. JAMA Intern Med, online 23. Januar 2017
$80 \%$ aller muslimischen Patienten mit Typ-2-Diabetes wollen während des Ramadan streng fasten. Das stellt für Patient und Arzt eine besondere Herausforderung dar. Muss die Therapie umgestellt werden? Kommt es zu Hypo- oder Hyperglykämien oder zu Gewichtsschwankungen? Bei Patienten, die bisher mit einem Sulfonylharnstoff behandelt werden, kann die Umstellung auf ein Therapieprinzip ohne Hypoglykämierisiko wie Gliptin oder GLP-1-Analogon in diesen Fällen sinnvoll sein.

Dr. med. Peter Stiefelhagen

- Herbsttagung der Deutschen Diabetes Gesellschaft, 11.11.2016 in Nürnberg

\section{Internet-Tipp}

Fremdsprachliche Schulungsmaterialien zum kostenlosen Download unter:

$\rightarrow$ http://migration.deutsche-diabetesgesellschaft.de/arbeitsmaterialien/materialien-in-fremdsprachen/diabetes-informationsmaterial-in-fremdsprachen.html $\rightarrow$ www.kbv.de/html/1150_20914.php 